\title{
Industry News
}

\section{Linkam Tensile Stage Chosen to Control Stress in Growth of Human Collagen by the University of Liverpool}

Linkam Scientific Instruments have been chosen by the Physics Department of the University of Liverpool to study the effects of controlling stresses applied during the growth of collagen secreted from human fibroblast cells. The research goal of senior research assistant, Dr. Caroline Smith, and her colleagues at the University of Liverpool is to advance the treatment of tendon injuries by developing a method of growing oriented human collagen.

Linkam Scientific Instruments Limited

www.linkam.co.uk

\section{California University Orders Vistec Electron Beam}

\section{Technology for Nano Research}

Vistec Lithography, Inc., a leading supplier of Electron Beam Lithography systems, has received an order for one of its EBPG5200 systems from the University of California, San Diego. The Vistec EBPG5200 will be installed in the Nano3 cleanroom facility at the California Institute for Telecommunications and Information Technology (Calit2) and will be used for nanoand micro-fabrication enabling basic and applied research and development for hundreds of academic and industrial users.

Vistec Lithography, Inc www.vistec-semi.com

\section{The OL 731 USB Smart Detector was Recently}

Featured on an Episode of the Discovery Channel's Mythbusters

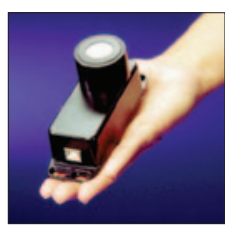

The segment entitled "Let There Be Light," which aired on the Wednesday, June 22nd episode, tested the theory of whether an underground chamber can be illuminated via light reflected off mirrors, much like a scene from the movie The Mummy. The OL 731 is a low-cost, hand-held radiometer/photometer that can be tailored to the spectral response of the user's specific application requirements.

Gooch \& Housego, formerly Optronic Laboratories www.goochandhousego.com/products/systems

\section{JEOL InTouchScope Scanning Electron Microscope Wins R\&D 100 Award}

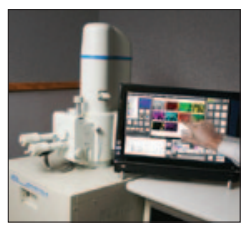

Having the familiar look of today's personal electronic media, the JEOL InTouchScope $\mathrm{e}^{\mathrm{TM}}$ SEM, a touchscreen-controlled analytical, portable low-vacuum Scanning Electron Microscope, has been recognized by the editors of R\&D Magazine as one of the 100 most technologically significant products introduced into the marketplace over the past year. It features integrated Energy Dispersive Spectroscopy (EDS), automated stage navigation capability, wireless capability, high- and low-vacuum operation, and a magnification range of $5 \times$ to $300,000 \times$.

JEOL USA, Inc.

www.jeolusa.com
Anasys Scientific Co-Founder Appointed to Prestigious Professorship in the College of Engineering at the University of Illinois, Urbana-Champaign

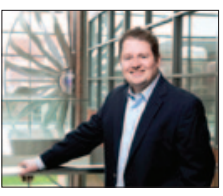

Anasys Instruments is pleased to announce that Dr. William P. King has been appointed as the Bliss Professor of Engineering at the University of Illinois at Urbana-Champaign. In addition to his faculty position at UIUC, Dr. King serves as a key scientific advisor to Anasys Instruments.

Anasys Instruments Corporation

www.anasysinstruments.com

\section{Peering Deeply Into the Surface}

Lehigh University is now home to two unique surfaceanalysis instruments that are the only such advanced surface characterization instruments in North America. The two new technologies will add significantly to national nanoscale surface science and analysis capabilities. Isolating and examining the very top or outer atomic layer, just 0.2 to $0.3 \mathrm{~nm}$ thick, can provide the most precise information about the nature of chemical reactions and why the phenomenon of nanotechnology is promising.

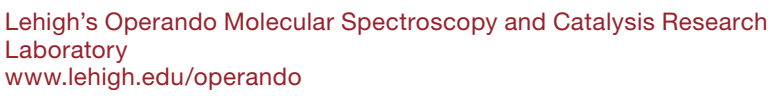

\section{FEI Provides 3D Analysis System to Whiting Oil and Gas Corporation}

FEI and Whiting Oil and Gas Corporation announce the purchase of QEMSCAN ${ }^{\circledR}$ automated petrography and Helios NanoLab ${ }^{\mathrm{TM}}$ DualBeam ${ }^{\mathrm{TM}}$ technology for Whiting's new rock lab in its Denver, Colorado headquarters. QEMSCAN provides data for reservoir characterization, including quantitative compositional and elastic properties critical for designing a successful hydraulic fracturing program. Given the intensive multi-rig drilling programs at Whiting, securing QEMSCAN technology in-house provides real-time solutions that can be applied prior to well completions.

FEl Company

www.fei-natural-resources.com

International Metallographic Society to

Appoint Buehler VP David Rollings

as Honorary CFO

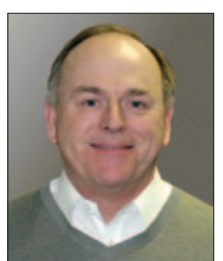

The International Metallographic Society (IMS), an affiliate society of ASM International, will appoint David Rollings as chief financial officer. The honorary position will be effective August 6 and announced at the 2011 Microscopy \& Microanalysis Conference, which IMS holds in conjunction with the Microscopy Society of America in Nashville, TN. As CFO, Rollings will be responsible for developing IMS's annual budget, making financial projections, and managing its corporate support program.

The International Metallographic Society, Inc www.internationalmetallographicsociety.org 
New 2011 Semrock Master Catalog

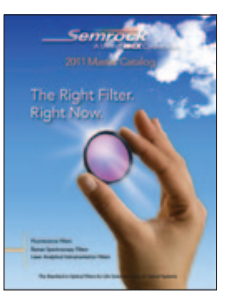

Semrock, Inc. announced the release of its new 100-page, full-color 2011 Master Catalog of optical filters and related products. This comprehensive catalog contains updated technical and product notes along with over 50 new hard-coated filter products for fluorescence microscopy and measurements, Raman spectroscopy, and other laser analytical instrumentation applications, as well as many improved versions of existing optical filters and filter sets. Services highlighted include our same-day shipping policy and rapid custom-sizing in under a week.

Semrock, Inc., a Unit of IDEX Corporation www.semrock.com

NanoWorld AG Launches High-Speed Scanning AFM Website

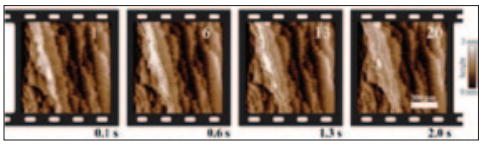

NanoWorld AG announced that it has launched a website entirely dedicated to High-Speed Scanning Atomic Force Microscopy (HS-AFM) at www.highspeedscanning. com. NanoWorld dedicates this website to the community of high-speed scanning AFM users and focuses on the probe aspect of high-speed scanning.

NanoWorld AG

www.highspeedscanning.com

\section{Canon Marketing Japan Expands to Support Growing} Industrial Markets with VersaXRM-500 from Xradia

Xradia, Inc. announced that Canon Marketing Japan has purchased the first VersaXRM-500. The recently announced VersaXRM-500 offers 3-D imaging with true spatial resolution at submicron levels. The VersaXRM features flexibility for correlative microscopy with multi-length scale imaging using the VersaXRM in conjunction with the UltraXRM or other microscopy techniques. The combination of larger working distance and imaging flexibility offers far superior performance advantage over traditional micro-CT systems.

Xradia, Inc.

www.xradia.com

Thermo Fisher Scientific Transforms and Simplifies Analysis of POPs with Pesticide Analyzer Reference Package

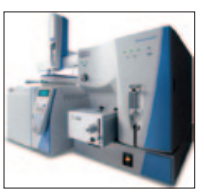

Thermo Fisher Scientific Inc. announced the second version of the Pesticide Analyzer Method Reference, a unique reference guide that simplifies the analysis of an expanded number of pesticides in environmental and food samples.

The new guide transforms the way pesticides and persistent organic pollutants (POPs), such as PCBs, PCDDs, and PCDFs, are analyzed. The newly revised, all-inclusive solution provides step-by-step information on using the QuEChERS extraction method.

Thermo Fisher Scientific Inc.

www thermofisher.com
Olympus Names Hidenao Tsuchiya Group

Vice President of Scientific Equipment

Business in the Americas

e 1 Olympus has named $\mathrm{Mr}$. Hidenao Tsuchiya as vice president and general manager of the Scientific Equipment Group in the Americas. Mr. Tsuchiya reports directly to Mr. Shin Nishigaki, president of the Life Science and Industrial Systems Group for Olympus Corporation in Japan. In this position, Mr. Tsuchiya replaces Mr. Osamu Joji, who has served as the group vice president and general manager for the last five years.

Olympus America Inc.

www.olympusamerica.com

\section{New Capture Interface for Image-Pro Software}

Media Cybernetics announces a new capture interface for Image-Pro software, supporting Princeton Instruments' MEGAPLUS industrial cameras. The interface is compatible with Windows XP Professional (SP3) 32- and 64-bit, Windows Vista Business or Ultimate (SP2) 32- and 64-bit, and Windows 7 (SP1) 32- and 64-bit operating systems. Supported cameras include ES 11000, ES 4020, and ES 2020 mono models; more to be supported soon. Download the interface on the Media Cybernetics support site at: http://support.mediacy.com/drivers.asp

Media Cybernetics www.mediacy.com

Asylum Research Appoints Dr. Mark Reitsma as Midwest U.S. Sales Director

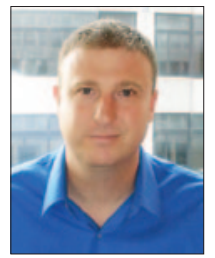

Asylum Research announced that it has appointed Dr. Mark Reitsma as its midwest U.S. sales director. Dr. Reitsma received his $\mathrm{PhD}$ in Engineering from the University of Newcastle, Australia for his work with AFM on geomaterials. For the past eight years, Mark has been working in the area of AFM force measurement calibration and investigation of materials properties at the U.S. National Institute of Standards and Technology (NIST).

Asylum Research

www.AsylumResearch.com

Olympus cellSens Version 1.5 Software

Supports Windows 7 with 64-Bit Operation;

Enhances Microscope Imaging and

Analysis

\section{OLYMPUS}

Olympus has launched the cellSens 1.5 software platform, the latest in its line of industry-leading applications for capturing and analyzing microscope images. cellSens 1.5 features Windows 764 -bit operation. It fully integrates all the imaging and analysis capabilities of such microscopes as the newly introduced fully motorized Olympus BX63 microscope, along with all previously supported Windows 7-compatible hardware and accessories.

Olympus America Inc.

www.olympusamerica.com/cellSens 